\title{
Prospects for Therapies in Osteoarthritis
}

\author{
Asim Ghouri ${ }^{1}$. Philip G. Conaghan ${ }^{1,2}$
}

Received: 14 November 2019 / Accepted: 6 February 2020 / Published online: 13 February 2020

(c) The Author(s) 2020

\begin{abstract}
Osteoarthritis (OA) is a chronic, debilitating disease affecting millions of people worldwide. Management of OA involves pharmacological and non-pharmacological approaches. Conventional pharmacological treatments have limited efficacy and are associated with a number of side-effects, restricting the number of patients who can use them. New pharmacological therapies for managing OA are required and a number have been developed targeting different tissues in OA: bone and cartilage, synovium and nerves. However, there has been overall limited success. Disease-modifying osteoarthritis drugs (DMOADs) are a putative class of therapies aimed at improving OA structural pathologies and consequent symptoms. Recent DMOAD studies have demonstrated some promising therapies but also provided new considerations for future trials.
\end{abstract}

Keywords Osteoarthritis $\cdot$ Nociceptive pain $\cdot$ DMOAD $\cdot$ Cartilage $\cdot$ Inflammation $\cdot$ Synovitis

\section{Introduction}

Osteoarthritis (OA) is a chronic, painful arthritis and a major cause of disability in affected individuals. At least 242 million people globally have hip/knee OA [1]. In addition, with an ageing population and rising risk factors such as obesity, this prevalence is growing [2]. As a result of the disability caused by OA, there is a significant cost to the global economy [3]. The pathogenesis of OA is complex with mechanical, genetic, metabolic and inflammatory pathways involved in its slowly evolving process [4]. Structure-pain relationships remain difficult to understand, and at the individual patient level, it is unclear which structures may be contributing to pain. Intra-articular candidates include bone and synovium, though it is worth noting that the amount of pain that pathologies in these tissues explain is small [5], and extra-articular features (e.g. tendinitis and bursitis) may confound associations.

Philip G. Conaghan

p.conaghan@leeds.ac.uk

1 Leeds Institute of Rheumatic and Musculoskeletal Medicine, University of Leeds and NIHR Leeds Biomedical Research Centre, Leeds, UK

2 Leeds Institute of Rheumatic and Musculoskeletal Medicine, Chapel Allerton Hospital, Chapeltown Rd, Leeds LS7 4SA, UK
OA management entails pharmacological and non-pharmacological approaches; when these fail to relieve symptoms, joint replacement surgery is considered. Non-pharmacological options (especially muscle strengthening) are often underutilised, and pharmacological options have not progressed significantly for decades: primarily paracetamol, non-steroidal anti-inflammatory drugs (NSAIDs, topical and oral) and opioids. However, the beneficial effect of paracetamol and opioids are limited, while NSAIDs and opioids are unsuitable for many patients because of their side-effects [6]. Intra-articular therapy, including corticosteroids, may also be used, although their effects tend to be short-lived.

This narrative review will update clinicians with recent developments on the use of existing pharmacological therapies and discuss novel treatments currently under an advanced stage of investigation (at least in phase II trials) for the treatment of OA. The therapies discussed have mostly been investigated for the treatment of knee OA and less commonly in hand OA. We have attempted to categorise these agents according to the tissues they target: bone and cartilage, nerves and synovium (the latter categorised as "immunomodulators"); we have included studies with both symptom and structural outcomes.

Though not systematic, this review was based on a PubMed search and review of meeting abstracts on trials reported between 2017 and 2019; both positive and negative reporting trials were included. We have excluded non-pharmacological treatments, therapies marketed as devices (e.g. 
hyaluronans) and nutraceuticals (e.g. glucosamine). Where relevant, older studies have been referenced to give context on the candidate therapy.

\section{Targeting Bone and Cartilage}

\section{Bisphosphonates}

Subchondral bone plays a role in maintaining hyaline articular cartilage integrity and its pathology is integral to the OA process [7]. A number of therapies used for osteoporosis have been explored as OA therapies, targeting altered bone turnover in the subchondral region [8,9]. Bisphosphonates have a long history of trials in OA, where they are thought to have a protective effect on subchondral bone and cartilage. A recent meta-analysis failed to demonstrate symptomatic improvement or reduction in radiographic OA progression with bisphosphonate therapy (Table 1) [10]. However, reduced MRI BML size was demonstrated in two studies. It is likely that many of the studies assessing radiographic progression were underpowered and many did not use modern inclusion criteria that enable detection of a pain response (such as pain $>4$ - on a 10-point scale).

Recent studies have focused recruitment on patients with definite subchondral bone abnormalities, specifically MRIdetected bone marrow lesions (BMLs). BMLs are commonly seen on MRI scans of OA knees, represent areas of trabecular loss, microfracture and fibrosis, and have associations with both pain and progressive cartilage loss [16-19]. Zoledronic acid (ZA) was compared with placebo in a doubleblind, parallel-group trial of 59 patients aged 50-80 with knee pain and at least one BML on MRI [13]. Results were promising, demonstrating a significant symptomatic benefit and reduction in BML size at 6 months. The preliminary report from the larger follow-up multicentre, randomised controlled trial has been recently presented. In this study, 223 knee OA patients with significant knee pain and MRIdetected BMLs received annual intravenous infusion of ZA $5 \mathrm{mg}$ or placebo over 2 years [20]. Unfortunately, no significant improvement was detected at 24 months in the Western Ontario and McMaster Universities Osteoarthritis Index (WOMAC) pain scale, WOMAC function scale, or BML size change with $\mathrm{ZA}$.

A sub-study of this 2-year ZA trial investigated the effects of ZA $5 \mathrm{mg}$ plus intravenous methylprednisolone $10 \mathrm{mg}$ in a preparation called VOLT0 ${ }^{17} .117$ patients with symptomatic knee OA were randomised to receive single dose VOLT01, ZA $5 \mathrm{mg}$ monotherapy or placebo. The study's primary outcome measurement was the incidence of the acute phase response following ZA administration (a flu-like or febrile response which can occur following IV bisphosphonate) and secondary outcomes measured BML size, WOMAC and visual analogue scale (VAS) pain and WOMAC function at 6 months. The incidence of acute phase responses was similar between treatment groups. In addition, VOLT01 and ZA were not superior to placebo in any of the secondary outcome pain measurements.

\section{Summary}

Recent studies have failed to demonstrate structural or symptomatic improvement with bisphosphonates in knee OA.

\section{Strontium Ranelate}

Strontium ranelate has also been trialled in knee OA. The SEKOIA trial investigated strontium 1 or $2 \mathrm{~g}$ /day versus placebo in a randomised, double-blind, 36-month study involving 1683 patients with symptomatic primary knee OA [21]. A significant reduction in radiographic joint space width (JSW; a surrogate for hyaline cartilage loss) reduction with both strontium doses compared to placebo was demonstrated at 36 months. The results also demonstrated a significant improvement in WOMAC total score and pain subscore at $2 \mathrm{~g} /$ day, though the effect size was very small (approximately 0.1 ). However, with a $14 \%$ annualised dropout rate, participant retention in (much desired) long-term OA trials is difficult and handling consequent missing data remains a complex issue. Strontium is also contraindicated in cardiovascular disease-a co-morbid condition patients with OA are also at increased risk of developing [22].

\section{Summary}

Strontium may improve pain and structural progression in knee OA but the benefits are small, and given potential cardiovascular risk, its use as a treatment is unlikely.

\section{Sprifermin}

Animal models of OA have demonstrated that fibroblast growth factor-18 can increase cartilage volume via proliferation of chondrocytes and modulating extracellular matrix turnover $[23,24]$. Sprifermin is a recombinant human fibroblast growth factor-18 (rhFGF18) which is administered intra-articularly and targets FGFR3 receptors in cartilage [25]. Sprifermin was investigated in a phase I, 1-year, randomised, double-blind, placebo-controlled, proof-of-concept trial at doses of $10 \mu \mathrm{g}, 30 \mu \mathrm{g}$, and $100 \mu \mathrm{g}$ [26]. Subjects received 2 cycles of three onceweekly injections (at weeks 0-2 and 13-15) of sprifermin or placebo and the results from 168 patients were evaluated. Although sprifermin failed to achieve its primary efficacy endpoint of significant reduction in loss of central medial femorotibial compartment cartilage thickness 


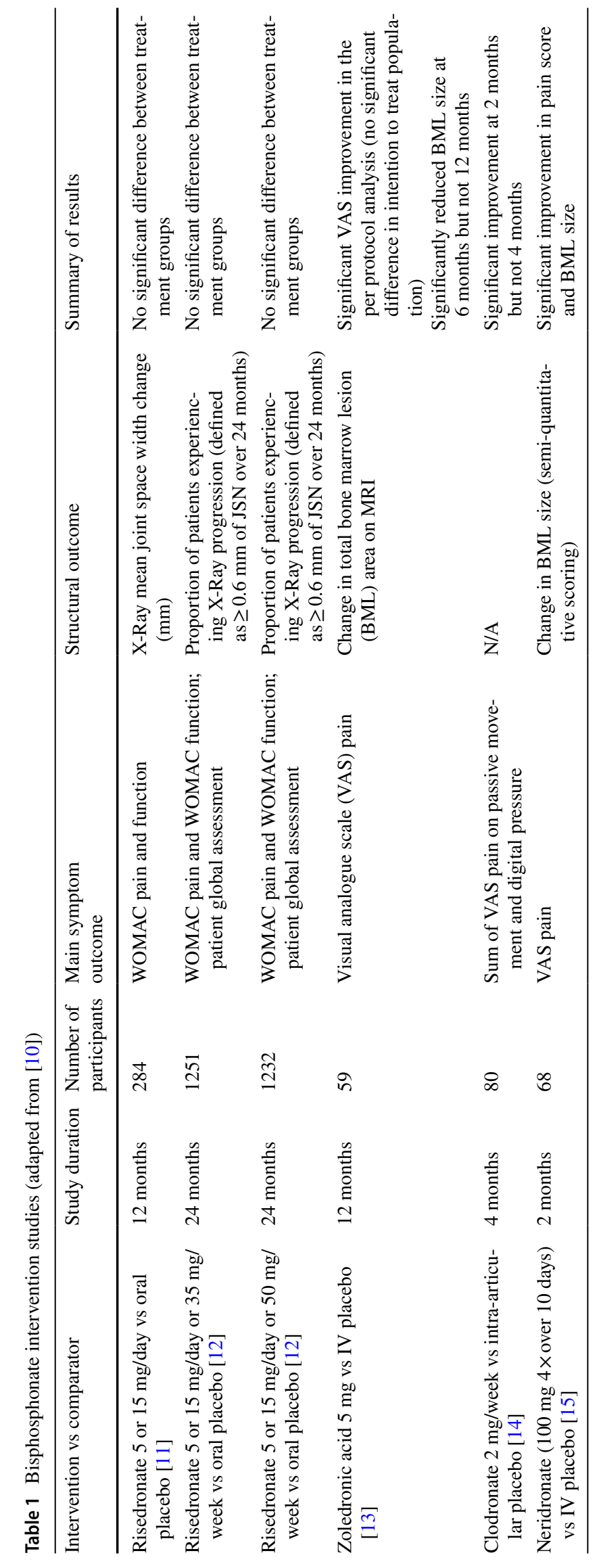


at 6 or 12 months, the study did, however, achieve its prespecified (and more sensitive) secondary structural efficacy MRI endpoints: significant reductions in loss of total femorotibial and lateral femorotibial cartilage thickness.

A further 5-year Phase II, dose-ranging, randomised trial of sprifermin (the FORWARD trial) is currently ongoing [27]. 549 patients were randomised to 1 of 5 groups: intra-articular injections of 100 microg of sprifermin administered 6-monthly $(n=110)$ or 12-monthly $(n=110) ; 30$ microg of sprifermin 6 -monthly $(n=111)$ or 12 -monthly $(n=110)$; or placebo every 6 months $(n=108)$. Each treatment cycle consisted of 3 onceweekly injections and total treatment duration was 18 months. Structural endpoints (cartilage thickness) were measured by quantitative MRI at the tibiofemoral joint. There was an initial dose-dependent increase in overall cartilage thickness with sprifermin $100 \mu \mathrm{g}$ for the first 2 years, though no significant improvement in WOMAC total score and pain, function, and stiffness subscale scores were detected between any of the treatment groups and placebo at 2 years [27].

Overall cartilage thickness reduced for all treatment groups between years 2 and 3 although the significant difference between sprifermin $100 \mu \mathrm{g}$ 6-monthly and placebo groups was maintained [28]. The loss of cartilage during the period may reflect the cessation of treatment. Preliminary results at 3 years also demonstrated a reduction in the expected (natural history) mean cartilage thickness loss from baseline to 3 years in patients on Sprifermin $100 \mu \mathrm{g} 6$-monthly versus placebo at the total femorotibial joint, and in the medial, lateral, central medial and central-lateral sub-regions. $18.4 \%$ (sprifermin) and 24.1\% (placebo) of participants discontinued the study within 3 years. At year 3 there was also no difference in symptom improvement between treatment groups, raising an important question regarding treatments improving $\mathrm{OA}$ structural pathologies: how long after structural change should patients be monitored in order to detect a difference in patient important outcomes (such as reduction in symptoms or joint replacement)?

The FORWARD study was primarily designed to assess structural progression and did not use modern pain trial inclusion criteria. Preliminary data from a post hoc analysis at year 3 of an 'at-risk' subgroup of patients with higher pain scores (WOMAC pain score of 40-90) and lower joint space width $(1.5-3.5 \mathrm{~mm})$ at baselinewhich are both predictors of more responsive outcome measurement-reported that patients with reduced medial or lateral joint space width at baseline along with higher baseline pain scores had significantly greater WOMAC pain score improvements with the highest dose of sprifermin (100 $\mu \mathrm{g} 6$-monthly) compared with placebo [29].

\section{Summary}

Sprifermin has demonstrated structural improvement in knee OA compared to placebo. Translation into symptomatic improvements is less certain and phase III trials are awaited.

\section{Wnt Pathway Inhibition}

The Wnt signalling pathway is a signal transduction pathway implicated in cartilage breakdown and OA pathophysiology, through its effect on chondrocyte, osteoblast and synovial cell differentiation [30, 31]. Altered expression of genes encoding Wnt signalling pathway proteins have been demonstrated in murine and human OA tissues, along with lower levels of the Wnt inhibitory protein DKK1 [31]. Lorecivivint (formerly SM04690) is an inhibitor of the Wnt signalling pathway and also inhibits the enzymes CDC-like kinase 2 (CLK2) and dual-specificity tyrosine phosphorylation-regulated kinase $1 \mathrm{~A}$, enhancing chondrogenesis, chondrocyte function and reducing inflammation [32]. It has been investigated in a phase IIa randomised, double-blind, placebocontrolled trial comparing 3 doses (low, medium and high) in 455 patients with knee OA. No statistically significant improvement in WOMAC A1 pain (pain when walking on a flat surface) was demonstrated between treatment groups and placebo. Exploratory analyses investigated the hypothesis that patients with bilateral symptomatic knee OA would be less responsive to treatment than patients with only one knee affected (a 'widespread pain' interference phenomenon). Two pre-specified subpopulations were therefore defined: unilateral symptomatic knee OA $(n=164)$ and subjects with unilateral knee OA without widespread pain $(n=128)$. Medium dose $(0.07 \mathrm{mg})$ SM04690 did significantly improve WOMAC A1 score versus placebo in unilateral symptomatic knee patients at 39 and 52 weeks and unilateral symptomatic patients without widespread pain at 26, 39 and 52 weeks [33]. Reduction in radiographic joint space width progression was also significantly greater in the $0.07 \mathrm{mg}$ treatment group versus placebo at week 52, though patient numbers were small for structural assessment [34].

A further 24-week, phase IIb study randomised 695 patients to receive a single dose lorecivivint $(0.03,0.07$, 0.15 or $0.23 \mathrm{mg}$ ), vehicle placebo or sham (dry needle only) at baseline. 635 subjects completed the study and the preliminary results demonstrated significant improvements from baseline in $0.07 \mathrm{mg}$ and $0.23 \mathrm{mg}$ lorecivivint dose groups compared to placebo for numeric rating scale pain (0.07 $\mathrm{mg}$ and $0.23 \mathrm{mg}$ at weeks 12 and week 24), WOMAC Pain $(0.07 \mathrm{mg}$ at week $12 ; 0.23 \mathrm{mg}$ at weeks 12 and 24), WOMAC Physical Function (0.07 mg at week $12 ; 0.23 \mathrm{mg}$ at weeks 12 and 24) and patient global assessment $(0.07 \mathrm{mg}$ at week 12;0.23 mg at weeks 12 and 24) [35]. In addition, a homogenous group of patients with joint space width 
2-4 $\mathrm{mm}$ and no widespread pain demonstrated an improved symptom effect size vs placebo at 12 and 24 weeks compared to the full analysis set at $0.07 \mathrm{mg}$ [36].

\section{Summary}

Sub-analyses of phase II trials suggest potential symptomatic and structural benefits with lorecivivint versus placebo. Await results of phase III trials which are underway.

\section{Cathepsin K Inhibition}

Cathepsin $\mathrm{K}$ is a cysteine protease highly expressed in activated osteoclasts. It plays an important role in bone resorption, degrading types I and II collagen and aggrecan found in cartilage. MIV-711 is a novel potent, selective and reversible inhibitor of cathepsin $\mathrm{K}$ which inhibits the actions of osteoclasts and is associated with reduced expression of biomarkers of bone resorption and cartilage loss [37]. Cathepsin K inhibition has also recently been shown to reduce the risk of fracture in post-menopausal osteoporosis, although with an increased cardiovascular risk [38]. A multicentre, randomised, placebo-controlled, double-blind, three-arm parallel, Phase IIa study evaluated the efficacy, safety and tolerability of MIV-711 in patients with knee OA [39]. Patients received oral MIV-711 $100 \mathrm{mg}, 200 \mathrm{mg}$ or matched placebo once daily for 26 weeks. No significant difference in pain reduction and quality of life scores were detected, although there was a trend towards increased reduction in pain scores with $100 \mathrm{mg}$ and $200 \mathrm{mg}$ doses at 26 weeks. There was a significant reduction in femoral OA bone disease progression on MRI at week 26 for MIV-711 $100 \mathrm{mg}$ and $200 \mathrm{mg}$ doses versus placebo and significant reduction in loss of cartilage thickness on the medial femur for $100 \mathrm{mg}$ dose versus placebo. A unique aspect of this trial was the key secondary outcome measure of 3D quantitative bone area using a machine learning method, enabling detection of change in a relatively small-number OA trial; this novel imaging biomarker can potentially be used as an outcome measure in further DMOAD trials [40]. The main adverse events reported were musculoskeletal symptoms, skin disorders and infections, although there was a reported overall acceptable safety profile [41]. Further studies are required to evaluate the potential structure-modifying effects of this agent.

\section{Summary}

Cathepsin $\mathrm{K}$ reduced knee OA structural progression but no significant symptom improvement over placebo was demonstrated over 6 months in a small phase II trial.

\section{Targeting Nerves}

As might be expected from this these modes of action these therapies are symptomatic and not trying to modify joint structure.

\section{Intra-articular Capsaicin}

Capsaicin is a chilli pepper extract which binds to a protein called transient receptor potential cation channel subfamily $\mathrm{V}$ member 1 (TRPV1), which is expressed on nociceptive nerve fibres (A $\delta$ and $\mathrm{C}$ ). It causes the burning sensation associated with chillies but is also considered an attractive target for potential analgesic medication, as its activation triggers a prolonged refractory state known as desensitisation [42]. CNTX-4975, the first intra-articular capsaicin preparation, is a highly purified, synthetic trans-capsaicin specifically targeting TRPV1-containing pain nociceptors [43]. Other sensory fibres such as touch or pressure are unaffected. Previous evidence supported topical capsaicin for relieving OA pain [44-47]. A phase II trial of single dose CNTX-4975 $1 \mathrm{mg}$ knee injection in patients with moderately painful knee OA was shown to improve the WOMAC A1 pain score (pain on walking) at weeks 12 and 24 in a 24-week, randomised, double-blind, placebo-controlled, dose-ranging study [48]. Data on adverse events are limited currently but the most common treatmentemergent adverse event is reported to be post-procedural pain, although this greatly subsides by $2 \mathrm{~h}$ post injection; in addition, there were no withdrawals due to side-effects [48]. Phase III trials of CNTX-4975 and a study examining the efficacy of repeated doses are currently in progress $[49,50]$.

\section{Summary}

Intra-articular capsaicin has demonstrated symptom improvement in knee OA. Phase III studies are in progress.

\section{Anti-nerve Growth Factor Monoclonal Antibodies}

Nerve growth factor (NGF) is a neurotrophin that binds to TrkA and has increased expression in OA [51]. It stimulates the growth of nociceptive nerve fibres and expression of nociceptive cell surface receptors. Osteoarthritic knee joints are highly innervated with nerve fibres in the joint capsule, ligaments, periosteum, menisci, subchondral bone and synovium [52]. This makes the peripheral nociceptive pathway an attractive target for novel analgesic agents.

\section{Tanezumab}

Tanezumab, and fasinumab are monoclonal antibodies targeting NGF, preventing it from binding its receptor to 
reduce pain [53]. Tanezumab is a humanised IgG type 2 monoclonal antibody produced by Pfizer and Eli Lilly with high specificity for NGF. Most data are available on tanezumab and in a meta-analysis of 9 studies with 10 randomised controlled trials enrolling 7665 patients with knee or hip OA, intravenous tanezumab $(2.5 \mathrm{mg}, 5 \mathrm{mg}$ and $10 \mathrm{mg}$ ) demonstrated superior efficacy compared to placebo/active comparator (significantly improving WOMAC pain subscale, WOMAC function subscale and patient global assessment) [54].

Fears of joint safety due to reported cases of rapidly progressive OA (RPOA) and then fears about possible sympathetic nerve problems (not borne out subsequently) meant this class of drugs underwent a number of regulatory holds, but are now back in development.

More recent studies have investigated subcutaneous (SC) preparations of tanezumab. A phase III trial of SC tanezumab compared fixed $(2.5 \mathrm{mg})$ doses 8 weeks apart and step up dosing ( $2.5 \mathrm{mg}$ administered at baseline, $5 \mathrm{mg}$ administered at week 8) versus placebo in 696 OA hip/ knee patients who had not responded to, or unable to tolerate, standard analgesia. Tanezumab $2.5 \mathrm{mg}$ fixed and $2.5 \mathrm{mg} / 5 \mathrm{mg}$ step up dosing was demonstrated to be superior to placebo in improving WOMAC Pain, WOMAC function and patient global assessment scores at week 16 [55]. There were more joint replacements observed in patients receiving tanezumab, although these were mostly elective and not associated with an AE. Two joint replacements were considered to be due to RPOA (see below).

Earlier phase III studies have demonstrated tanezumab monotherapy at $5 \mathrm{mg}$ and $10 \mathrm{mg}$ to have greater analgesic efficacy over NSAIDs (celecoxib $100 \mathrm{mg}$ and naproxen $500 \mathrm{mg}$ ) and oxycodone $10-40 \mathrm{mg}[56,57]$. Combination tanezumab and NSAID therapy also demonstrated significantly greater analgesic efficacy over NSAID monotherapy but not compared with tanezumab monotherapy [57]. A recent phase III study investigated SC tanezumab $2.5 \mathrm{mg}$ or $5 \mathrm{mg}$ versus NSAID (naproxen $500 \mathrm{mg}$, celecoxib $100 \mathrm{mg}$, or diclofenac ER $75 \mathrm{mg}$ orally) in an 80-week study of 2996 patients with hip or knee OA. Tanezumab $5 \mathrm{mg}$ significantly improved WOMAC pain and WOMAC function scores compared to the NSAID group at week 16 but not the Patient's Global Assessment of OA. There were no significant improvements compared to NSAID with tanezumab $2.5 \mathrm{mg}$ at 16 weeks or with either tanezumab doses at 56 weeks [58].

Tanezumab (as the most widely studied drug) has been associated with a number of adverse effects, although discontinuation rates in anti-NGF trials are low [54]. Reported side-effects include paraesthesia, headaches, arthralgia, peripheral oedema, peripheral neuropathy, hypo- and hyper- aesthesia. Lower doses of tanezumab are associated with fewer adverse events [54]. Arthralgia was the most commonly reported side effect ( $8 \%$ of tanezumab-treated patients).

\section{Fasinumab}

Fasinumab is also a humanised monoclonal antibody developed by Regeneron and Teva with high specificity and affinity for NGF. It was recently investigated in a phase IIb/ III double-blind, placebo-controlled, randomised clinical trial of 421 patients with moderate-to-severe knee or hip $\mathrm{OA}$ and inadequate response or intolerance to analgesics [59]. Patients were randomised to receive fasinumab $1 \mathrm{mg}$, $3 \mathrm{mg}, 6 \mathrm{mg}, 9 \mathrm{mg}$ or placebo every 4 weeks over 16 weeks with follow-up until week 36.346 patients completed the study. Statistically and clinically significant improvements in WOMAC pain, WOMAC physical function subscale and patient global assessment scores were detected with all the doses of fasinumab compared to placebo at week 16 . These improvements were not dose-dependent.

\section{Rapidly progressive $O A$}

Rapidly progressive OA (RPOA) is the most serious adverse event reported with the anti-NGF therapies, with the risk apparently dose-related [59, 60]. It is a painful condition diagnosed radiographically by rapid joint space narrowing and severe progressive atrophic bone [61]. Recent trials have used a maximal $5 \mathrm{mg}$ dose of tanezumab as the RPOA risk is reduced and outweighed by its potential therapeutic benefit [62]. Although a higher rate of RPOA is observed with tanezumab $2.5 \mathrm{mg}$ and $5 \mathrm{mg}$ compared to NSAID [63], the combination of tanezumab and NSAIDs appears to increase the risk of RPOA compared to tanezumab alone [62], and modern trials have also been designed to reduce concomitant NSAID use.

\section{Summary}

Anti-NGF antibodies have demonstrated symptomatic improvement in hip and knee OA. Higher doses increase the risk of developing RPOA. These agents may be the first new OA therapy to emerge in many years, though careful evaluation of benefit-risk will be required.

\section{Immunomodulators}

\section{Injectable Corticosteroids}

Intra-articular corticosteroids have been shown to be effective in reducing OA pain, although their effects are short lived with no associated benefit seen at 6 months [64]. A Cochrane review of 27 trials investigating intra-articular 
corticosteroids in knee OA found an association with small to moderate improvement in function at up to 6 weeks, but no improvement beyond this timeframe [65]. Moderate to large heterogeneity between trials was also found.

\section{Intra-articular Triamcinolone Acetonide Extended Release}

Given the reported short-term benefits of intra-articular corticosteroid, FX006, a triamcinolone acetonide extended release (TA-ER) formulation produced using microsphere technology, with the aim of giving prolonged benefits, was investigated in patients with knee OA. TA-ER at doses $10 \mathrm{mg}, 40 \mathrm{mg}$ and $60 \mathrm{mg}$ were compared in a phase IIa randomised, double-blind, controlled, dose-finding trial [66]. 228 patients with knee OA were followed up for 12 weeks post single intra-articular knee injection. There was a significant improvement in mean daily pain intensity scores (the primary outcome measure) with TA-ER $40 \mathrm{mg}$ versus immediate-release triamcinolone $40 \mathrm{mg}$ at weeks 5-10; furthermore, all WOMAC subscale scores were significantly improved with TA-ER $40 \mathrm{mg}$ compared to immediaterelease triamcinolone at 8 weeks. No superiority was demonstrated with TA-ER $10 \mathrm{mg}$ and $60 \mathrm{mg}$ versus immediaterelease triamcinolone $40 \mathrm{mg}$ in improving mean daily pain intensity, although TA-ER $10 \mathrm{mg}$ was significantly superior to immediate-release triamcinolone $40 \mathrm{mg}$ in improving some secondary efficacy endpoints. Similar frequencies of adverse events (AEs) were reported between TA-ER and immediate-release triamcinolone.

TA-ER was compared to placebo in a further phase IIB study of 306 knee OA patients. The primary outcome of a significant improvement in average daily pain (ADP) intensity versus placebo at 12 weeks was not achieved; however, TA-ER $32 \mathrm{mg}$ significantly improved ADP intensity scores versus placebo at weeks $1-11$ and at week 13 [67]. More recently, a phase III, multicentre, doubleblinded, randomised, controlled trial comparing TA-ER $32 \mathrm{mg}$ to immediate-release triamcinolone $40 \mathrm{mg}$ and placebo in 484 knee OA patients achieved its primary endpoint of a significant improvement in ADP intensity compared to placebo at 12 weeks [68]. TA-ER did not significantly improve ADP intensity over immediaterelease triamcinolone at 12 weeks; however, WOMAC pain, stiffness and physical function scores, and the Knee injury and Osteoarthritis Outcome Score (Quality of Life subdomain-KOOS-QOL) at 12 weeks were significantly improved with TA-ER $32 \mathrm{mg}$ compared to both placebo and immediate-release triamcinolone. The differences with the active comparator seen when using different outcome measures suggests a greater responsiveness for the disease-specific, multi-item WOMAC tool over the ADP single item question. As TA-ER has been shown to be more effective than placebo, it has been licensed by the FDA for managing OA-related knee pain. A further advantage of TA-ER's mechanism of action is reduced systemic exposure compared to immediate-release triamcinolone, due to its slower intra-articular release [69]. TA-ER $32 \mathrm{mg}$ has been shown to have less effect on glycaemic control than standard triamcinolone $40 \mathrm{mg}$ in type 2 diabetic patients [70].

\section{Summary}

Intramuscular and intra-articular steroid provides short-lived symptomatic benefit in OA. An extended release intra-articular steroid is now licensed in the USA.

\section{Hydroxychloroquine}

Hydroxychloroquine is routinely used in the management of rheumatoid arthritis synovitis and has also been used in the management of inflammatory hand $\mathrm{OA}$, related to anecdotal evidence of benefit and a tolerable safety profile [71, 72]. The immunomodulatory actions of hydroxychloroquine work through inhibitory effects on toll-like receptor (TLR) signalling [73]. This was considered potentially therapeutic in OA as TLRs are upregulated in OA cartilage tissue and trigger its breakdown via pro-inflammatory pathways [74, 75]. Furthermore, there is evidence of synovitis in hand OA $[76,77]$. Early small pilot studies suggested symptomatic improvement following hydroxychloroquine treatment [78, 79]. A large randomised, double-blind, placebo-controlled clinical trial investigated this further in 248 patients over a 12-month period [80]. Patients with moderate-to-severe hand pain were randomised to hydroxychloroquine or placebo, along with their usual analgesic medication. No significant reduction in hand pain with additional hydroxychloroquine compared to placebo at 6 months was detected, therefore not achieving primary endpoint. Hydroxychloroquine also did not slow radiographic OA progression compared to placebo at 12 months. A subset of patients who were stratified for (commonly found) ultrasound-detected synovitis also did not demonstrate hydroxychloroquine efficacy. Another randomised controlled trial compared hydroxychloroquine $400 \mathrm{mg}$ to placebo in 196 patients with hand OA (but not on concomitant NSAID or corticosteroid treatment) and did not detect a significant difference in pain scores after 24 weeks of treatment [81].

\section{Summary}

Latest evidence demonstrates hydroxychloroquine is ineffective in reducing patient symptoms in hand OA. 


\section{Tumour Necrosis Factor Inhibitors}

Evidence suggests that tumour necrosis factor alpha (TNF $\alpha)$ is implicated in OA pathogenesis [82]. However, studies of adalimumab have not shown it to be effective compared to placebo in reducing symptoms of hand OA [83, 84]. More recently, the HUMOR trial compared subcutaneous adalimumab $40 \mathrm{mg}$ on alternative weeks versus placebo over 12 weeks in a crossover-design study of 43 patients with erosive hand $\mathrm{OA}$ and synovitis seen on magnetic resonance imaging (MRI) [85]. There was an 8-week washout period before treatment groups crossed over. Adalimumab did not significantly reduce visual analogue scale (VAS) scores compared to placebo. In addition, there were no significant differences detected for secondary outcome measures including change in MRI-detected synovitis and bone marrow lesions.

Another anti-TNF $\alpha$ agent, etanercept, was recently studied in a 1-year, double-blind, randomised, placebo-controlled, multicentre trial of 90 patients with symptomatic erosive inflammatory hand OA. The primary endpoint of a significant improvement in VAS pain at 24 weeks was not achieved with etanercept $50 \mathrm{mg}$ weekly [86]. In addition, there was no significant treatment reduction in ultrasonographic or MRI-detected synovitis after 1 year. A significant reduction in MRI-detected bone marrow lesions in the interphalangeal joints of one hand was detected after 1 year with etanercept; however, this was in a very small subgroup ( $\mathrm{n}=10$ in each treatment group).

\section{Summary}

Anti-TNF agents have not demonstrated symptomatic improvement versus placebo in OA.

\section{Interleukin-1 $\alpha$ and $\beta$ Inhibition}

Interleukin- 1 alpha (IL-1 $\alpha$ ) and interleukin- 1 beta (IL-1 $\beta$ ) expression is increased within OA cartilage and synovial membrane [87, 88]. Raised IL-1 levels are also associated with increased expression of OA pathophysiology markers in affected fluids and tissue including catabolic enzymes, prostaglandins, nitric oxide and other markers [89]. Blockage of the interleukin-1 receptor has been shown to slow the progression of OA in animal models [90-93].

Anakinra, a recombinant form of interleukin-1 receptor antagonist (IL-1Ra), was investigated in a randomised, multicentre, double-blind, placebo-controlled study of 170 knee OA patients who received a single intra-articular injection of placebo, anakinra $50 \mathrm{mg}$, or anakinra $150 \mathrm{mg}$ in their symptomatic knee. No significant difference in the mean
WOMAC pain score improvements from baseline to week 4 could be detected between the treatment groups, although anakinra was well tolerated [94].

Lutikizumab (formerly ABT-981) is a novel human dual variabledomain immunoglobulin that targets and inhibits IL- $1 \alpha$ and IL- $1 \beta$ [95]. This was investigated in the ILLUSTRATE-K trial, a randomised, double-blind, placebo-controlled, parallel-group phase II study comparing fortnightly subcutaneous injections of lutikizumab at $25 \mathrm{mg}, 100 \mathrm{mg}$, or $200 \mathrm{mg}$ in patients with knee OA over 50 weeks [96]. The study achieved its primary endpoint with preliminary results demonstrating a significant improvement in WOMAC pain score at 16 weeks with lutikizumab $100 \mathrm{mg}$ compared to placebo. However, there was no significant improvement with lutikizumab $25 \mathrm{mg}$ or $200 \mathrm{mg}$ compared to placebo at this time point. Cartilage thickness, MRI synovitis, and other structural endpoints were similar between lutikizumab and placebo, although lutikizumab was generally well tolerated. With the lack of dose response and failure to meet structural endpoints, there is uncertainty regarding the clinical efficacy of lutikizumab in knee OA based on this study.

Lutikizumab was also trialled in patients with erosive hand OA, in a phase IIa, placebo-controlled, randomised study. Clinical and radiological outcomes were measured in 131 patients with hand OA as per ACR criteria ( $\geq 3$ inflamed interphalangeal joints which are tender, swollen, or both, hand pain $\geq 6$ (scale $0-10$ ), and $\geq 1$ erosive interphalangeal joint on X-ray). Subjects were given lutikizumab $200 \mathrm{mg}$ $(n=67)$ or placebo $(n=64)$ every 2 weeks for 26 weeks. Preliminary data did not demonstrate a significant improvement in Australian/Canadian Hand OA Index (AUSCAN) pain scores with lutikizumab compared to placebo at 16 weeks. There was also no significant difference in X-Ray or MRI data between treatment groups [97].

However, there have been further developments in the use of IL-1 in treating OA, involving canakinumab, a monoclonal antibody targeting IL-1 $\beta$. Previous in vitro studies of canakinumab on human chondrocytes demonstrated increased proteoglycan and reduced nitric oxide synthesis which may reduce cartilage breakdown [98]. Canakinumab was recently studied in the CANTOS trial: a very large randomised, placebo-controlled trial investigating the cardiovascular effect of subcutaneous canakinumab [99]. 10,061 patients with previous myocardial infarction and a high-sensitivity C-reactive protein level $\geq 2 \mathrm{mg} / \mathrm{L}$ on blood testing were given canakinumab doses of $50 \mathrm{mg}, 150 \mathrm{mg}$ or $300 \mathrm{mg}$ every 3 months for a median of 3.7 years. The study demonstrated that canakinumab at a dose of $150 \mathrm{mg}$ was associated with a significantly lower rate of recurrent cardiovascular events compared to placebo, independent of lipid-level lowering. Despite this being primarily a cardiovascular study, a post hoc study reported reduced incidence of OA symptoms (reported as adverse events) and total knee 
and hip replacements in the patients who received canakinumab [100]. The implications of these findings are unclear.

\section{Summary}

Although previous studies of IL-1 inhibition in OA did not demonstrate a symptomatic or structural benefit, recent analyses from the CANTOS study suggest benefits in reducing joint replacement; more research in this area is required.

\section{Methotrexate}

Methotrexate has been investigated in a phase III trial of patients with knee OA, targeting the synovitis and associated pain in the condition [101]. 155 patients were randomised to receive methotrexate (dose escalating 10-25 mg over 8 weeks with maintenance dose at $25 \mathrm{mg}$ or the highest tolerated dose) or placebo. Preliminary data demonstrated statistically significant improvements with methotrexate at 6 months for WOMAC stiffness and physical function, but not pain. This benefit was reduced at 12 months. As a secondary outcome measure, synovial volume was measured on MRI at baseline and 6 months with no significant difference between treatment groups.

Methotrexate has also been investigated in patients with erosive hand OA [102]. 64 patients were randomised to receive methotrexate $10 \mathrm{mg}$ weekly or placebo. There was no significant difference between treatment groups in VAS pain score reduction at 3 months; therefore the study did not achieve its primary outcome. There was also no significant improvement in pain at 12 months; however, there appeared to be less structural progression in the methotrexate group, with more remodelling seen in erosive joints.

\section{Summary}

Usual dose methotrexate has demonstrated symptomatic improvement at 6 months in knee OAbut low dose has not been beneficial in hand OA. More consideration of methotrexate benefits are required as a potential analgesic therapy.

\section{Conclusion}

Treatment options for osteoarthritis pain remain limited. Different joint structures have been targeted in pharmacological intervention studies of OA with variable levels of success. Despite structural improvement with a cartilage anabolic agent and an osteoclast inhibitor, their effect on symptoms are still under investigation. The limitations in developing novel therapeutics in OA are partly related to our limited understanding of the structure-pain relationship in $\mathrm{OA}$, resulting in a current lack of attractive tissue target in
OA. However, recent trial data of agents targeting peripheral nociceptive pathways in knee OA have been promising.

Funding This work was supported in part by the Versus Arthritis Experimental Osteoarthritis Treatment Centre (ref 20083) and the National Institute for Health Research (NIHR) through the Leeds Biomedical Research Centre. The views expressed are those of the authors and not necessarily those of the NHS, the NIHR or the Department of Health.

\section{Compliance with Ethical Standards}

Conflict of interest AG reports no conflicts. PGC reports speakers' bureaus or consultancies for Abbvie, Astra Zeneca, BMS, Centrexion, EMD Serono, Flexion Therapeutics, Galapagos, GlaxoSmithKline, Novartis, Pfizer, Samumed and Stryker.

Human and Animal Rights and Informed Consent This article does not contain any studies with human participants or animals performed by any of the authors.

Open Access This article is licensed under a Creative Commons Attribution 4.0 International License, which permits use, sharing, adaptation, distribution and reproduction in any medium or format, as long as you give appropriate credit to the original author(s) and the source, provide a link to the Creative Commons licence, and indicate if changes were made. The images or other third party material in this article are included in the article's Creative Commons licence, unless indicated otherwise in a credit line to the material. If material is not included in the article's Creative Commons licence and your intended use is not permitted by statutory regulation or exceeds the permitted use, you will need to obtain permission directly from the copyright holder. To view a copy of this licence, visit http://creativecommons.org/licenses/by/4.0/.

\section{References}

1. C Global Burden of Disease Study (2015) Global, regional, and national incidence, prevalence, and years lived with disability for 301 acute and chronic diseases and injuries in 188 countries, 1990-2013: a systematic analysis for the Global Burden of Disease Study 2013. Lancet (London, England) 386(9995):743-800

2. Neogi T, Zhang Y (2013) Epidemiology of osteoarthritis. Rheum Dis Clin N Am 39(1):1-19

3. Salmon JH et al (2016) Economic impact of lower-limb osteoarthritis worldwide: a systematic review of cost-of-illness studies. Osteoarthr Cartil 24(9):1500-1508

4. Chen D et al (2017) Osteoarthritis: toward a comprehensive understanding of pathological mechanism. Bone Res 5:16044

5. Yusuf E et al (2011) Do knee abnormalities visualised on MRI explain knee pain in knee osteoarthritis? A systematic review. Ann Rheum Dis 70(1):60

6. Zhang W et al (2010) OARSI recommendations for the management of hip and knee osteoarthritis: part III: changes in evidence following systematic cumulative update of research published through January 2009. Osteoarthr Cartil 18(4):476-499

7. Sharma AR et al (2013) Interplay between cartilage and subchondral bone contributing to pathogenesis of osteoarthritis. Int J Mol Sci 14(10):19805-19830 
8. Blumenkrantz G et al (2004) A pilot, two-year longitudinal study of the interrelationship between trabecular bone and articular cartilage in the osteoarthritic knee. Osteoarthr Cartil 12(12):997-1005

9. Karvonen RL et al (1998) Periarticular osteoporosis in osteoarthritis of the knee. J Rheumatol 25(11):2187-2194

10. Vaysbrot EE et al (2018) Are bisphosphonates efficacious in knee osteoarthritis? A meta-analysis of randomized controlled trials. Osteoarthr Cartil 26(2):154-164

11. Spector TD et al (2005) Effect of risedronate on joint structure and symptoms of knee osteoarthritis: results of the BRISK randomized, controlled trial [ISRCTN01928173]. Arthritis Res Ther 7(3):R625-R633

12. Bingham CO 3rd et al (2006) Risedronate decreases biochemical markers of cartilage degradation but does not decrease symptoms or slow radiographic progression in patients with medial compartment osteoarthritis of the knee: results of the two-year multinational knee osteoarthritis structural arthritis study. Arthritis Rheum 54(11):3494-3507

13. Laslett LL et al (2012) Zoledronic acid reduces knee pain and bone marrow lesions over 1 year: a randomised controlled trial. Ann Rheum Dis 71(8): 1322

14. Rossini M et al (2015) Effects of intra-articular clodronate in the treatment of knee osteoarthritis: results of a double-blind, randomized placebo-controlled trial. Rheumatol Int 35(2):255-263

15. Varenna $M$ et al (2015) Intravenous neridronate in the treatment of acute painful knee osteoarthritis: a randomized controlled study. Rheumatology (Oxford) 54(10):1826-1832

16. Felson DT et al (2001) The association of bone marrow lesions with pain in knee osteoarthritis. Ann Intern Med 134(7):541-549

17. Zhai $\mathrm{G}$ et al (2006) Correlates of knee pain in older adults: Tasmanian older adult cohort study. Arthritis Care Res 55(2):264-271

18. Felson DT et al (2003) Bone marrow edema and its relation to progression of knee osteoarthritis. Ann Intern Med 139(5_Part_1):330-336

19. Castañeda $\mathrm{S}$ et al (2012) Subchondral bone as a key target for osteoarthritis treatment. Biochem Pharmacol 83(3):315-323

20. Cai $\mathrm{G}$ et al (2018) OP0016 A multicentre randomised controlled trial of zoledronic acid for osteoarthritis of the knee with bone marrow lesions. Ann Rheum Dis 77(Suppl 2):57

21. Reginster J-Y et al (2013) Efficacy and safety of strontium ranelate in the treatment of knee osteoarthritis: results of a doubleblind, randomised placebo-controlled trial. Ann Rheum Dis 72(2): 179

22. Wang $\mathrm{H}$ et al (2016) Osteoarthritis and the risk of cardiovascular disease: a meta-analysis of observational studies. Sci Rep 6:39672-39672

23. Moore EE et al (2005) Fibroblast growth factor-18 stimulates chondrogenesis and cartilage repair in a rat model of injuryinduced osteoarthritis. Osteoarthr Cartil 13(7):623-631

24. Reker D et al (2017) Sprifermin (rhFGF18) modulates extracellular matrix turnover in cartilage explants ex vivo. J Transl Med 15(1):250

25. Davidson D et al (2005) Fibroblast growth factor (FGF) 18 signals through FGF receptor 3 to promote chondrogenesis. J Biol Chem 280(21):20509-20515

26. Lohmander LS et al (2014) Intraarticular sprifermin (recombinant human fibroblast growth factor 18) in knee osteoarthritis: a randomized, double-blind, placebo-controlled trial. Arthritis Rheumatol 66(7):1820-1831

27. Hochberg MC et al (2019) Effect of intra-articular sprifermin vs placebo on femorotibial joint cartilage thickness in patients with osteoarthritis: the FORWARD Randomized Clinical Trial. JAMA 322(14):1360-1370
28. Hochberg M et al (2018) OP0059 Efficacy and safety of intraarticular sprifermin in symptomatic radiographic knee osteoarthritis: pre-specified analysis of 3-year data from a 5-year randomised, placebo-controlled, phase ii study. Ann Rheum Dis 77(Suppl 2):80-81

29. Gühring H et al (2019) OP0010 cartilage thickness modification with sprifermin in knee osteoarthritis patients translates into symptomatic improvement over placebo in patients at risk of further structural and symptomatic progression: post-hoc analysis of the phase II forward trial. Ann Rheum Dis 78(Suppl 2):70

30. Deshmukh V et al (2018) A small-molecule inhibitor of the Wnt pathway (SM04690) as a potential disease modifying agent for the treatment of osteoarthritis of the knee. Osteoarthr Cartil 26(1):18-27

31. Zhou Y et al (2017) Wnt/ $\beta$-catenin signaling in osteoarthritis and in other forms of arthritis. Curr Rheumatol Rep 19(9):53-53

32. Deshmukh V et al (2019) Modulation of the Wnt pathway through inhibition of CLK2 and DYRK1A by lorecivivint as a novel, potentially disease-modifying approach for knee osteoarthritis treatment. Osteoarthr Cartil 27(9):1347-1360

33. Kennedy S et al (2018) OP0061 Treatment of knee osteoarthritis with sm04690 improved womac a1 'pain on walking'-results from a 52 week, randomised, double-blind, placebo-controlled, phase 2 study of a novel, intra-articular, wnt pathway inhibitor. Ann Rheum Dis 77(Suppl 2):81-82

34. Yazici Y et al (2018) Radiographic outcomes were concordant with outcome measures in rheumatology-osteoarthritis research society international (OMERACT-OARSI) strict response: post-hoc analysis from a phase 2 study of a WNT pathway inhibitor, SM04690, for knee osteoarthritis treatment. Osteoarthr Cartil 26:S244-S245

35. Yazici Y et al (2019) THU0458 Efficacy and safety from a phase 2B trial of SM04690, a novel intra-articular wnt pathway inhibitor for the treatment of osteoarthritis of the knee. Ann Rheum Dis 78(Suppl 2):519

36. Kennedy S et al (2019) Subject enrichment criteria for phase 3 studies of lorecivivint (SM04690), a potential disease-modifying knee osteoarthritis drug: a Post Hoc Study on the effects of baseline comorbid pain and joint space width on patientreported outcomes [abstract]. Arthritis Rheumatol 71(suppl 10). http://acrabstracts.org/abstract/subject-enrichment-crite ria-for-phase-3-studies-of-lorecivivint-sm04690-a-poten tial-disease-modifying-knee-osteoarthritis-drug-a-post-hocstudy-on-the-effects-of-baseline-comorbid-pain-and-joint-s/. Accessed Dec 242019

37. Lindström E et al (2018) Nonclinical and clinical pharmacological characterization of the potent and selective cathepsin K inhibitor MIV-711. J Transl Med 16(1):125

38. McClung MR et al (2019) Odanacatib for the treatment of postmenopausal osteoporosis: results of the LOFT multicentre, randomised, double-blind, placebo-controlled trial and LOFT Extension study. Lancet Diabetes Endocrinol 7(12):899-911

39. Conaghan P et al (2017) Miv-711, a novel Cathepsin K inhibitor demonstrates evidence of osteoarthritis structure modification: results from a 6 month randomized double-blind placebo-controlled phase IIA trial [abstract]. Arthritis Rheumatol 69(suppl 10). http://acrabstracts.org/abstract/miv-711-a-novel -cathepsin-k-inhibitor-demonstrates-evidence-of-osteoarthr itis-structure-modification-results-from-a-6-month-randomized -double-blind-placebo-controlled-phase-iia-trial/. Accessed Dec 242019

40. Bowes MA et al (2015) A novel method for bone area measurement provides new insights into osteoarthritis and its progression. Ann Rheum Dis 74(3):519

41. Conaghan PG et al (2018) Six months' treatment with MIV711, a novel Cathepsin $\mathrm{K}$ inhibitor induces osteoarthritis 
structure modification: results from a randomized doubleblind placebo-controlled phase IIA trial. Osteoarthr Cartil 26:S25-S26

42. Szallasi A et al (2007) The vanilloid receptor TRPV1: 10 years from channel cloning to antagonist proof-of-concept. Nat Rev Drug Discov 6:357

43. Centrexion Therapeutics (2018) A novel approach to treating pain. https://centrexion.com/our-pipeline/. Accessed $13 \mathrm{Sept}$ 2018

44. Altman RD et al (1994) Capsaicin cream $0.025 \%$ as monotherapy for osteoarthritis: a double-blind study. Semin Arthritis Rheum 23(6):25-33

45. Mason L et al (2004) Systematic review of topical capsaicin for the treatment of chronic pain. BMJ 328(7446):991

46. Zhang WY, Li-Wan-Po A (1994) The effectiveness of topically applied capsaicin. A meta-analysis. Eur J Clin Pharmacol 46:517-522

47. Kosuwon W et al (2010) Efficacy of symptomatic control of knee osteoarthritis with $0.0125 \%$ of capsaicin versus placebo. J Med Assoc Thail 93:1188-1195

48. Stevens RM et al (2019) Randomized, double-blind, placebocontrolled trial of intra-articular CNTX-4975 (trans-capsaicin) for pain associated with osteoarthritis of the knee. Arthritis Rheumatol 71(9):1524-1533

49. Stevens R (2018) A clinical study to test efficacy and safety of CNTX-4975-05 in patients with osteoarthritis knee pain. 2018 September 07, 2018. https://clinicaltrials.gov/ct2/show/NCT03 429049? $\mathrm{id}=\mathrm{NCT} 02508155+\mathrm{OR}+\mathrm{NCT} 03660943+\mathrm{OR}+\mathrm{NCT} 03$ 429049\&rank=2\&load=cart. Accessed 10 Sept 2018

50. Stevens R (2018) A Clinical study to test efficacy and safety of repeat doses of CNTX-4975-05 in patients with osteoarthritis knee pain. https://clinicaltrials.gov/ct2/show/NCT0366094 3 ? $\mathrm{id}=\mathrm{NCT} 02508155+\mathrm{OR}+\mathrm{NCT} 03660943+\mathrm{OR}+\mathrm{NCT} 0342904$ 9\&rank $=1 \&$ load $=$ cart. Accessed 7 Sept 2018

51. Denk F, Bennett DL, McMahon SB (2017) Nerve growth factor and pain mechanisms. Annu Rev Neurosci 40(1):307-325

52. Malfait A-M, Schnitzer TJ (2013) Towards a mechanism-based approach to pain management in osteoarthritis. Nat Rev Rheumatol 9:654

53. Abdiche YN, Malashock DS, Pons J (2009) Probing the binding mechanism and affinity of tanezumab, a recombinant humanized anti-NGF monoclonal antibody, using a repertoire of biosensors. Protein Sci 17(8):1326-1335

54. Chen J et al (2017) Efficacy and safety of tanezumab on osteoarthritis knee and hip pains: a meta-analysis of randomized controlled trials. Pain Med 18(2):374-385

55. Schnitzer TJ et al (2019) Effect of tanezumab on joint pain, physical function, and patient global assessment of osteoarthritis among patients with osteoarthritis of the hip or knee: a randomized clinical trialeffect of tanezumab on joint pain, physical function, and patient global assessment of osteoarthritiseffect of tanezumab on joint pain, physical function, and patient global assessment of osteoarthritis. JAMA 322(1):37-48

56. Spierings ELH et al (2013) A phase III placebo- and oxycodonecontrolled study of tanezumab in adults with osteoarthritis pain of the hip or knee. PAIN® 154(9):1603-1612

57. Schnitzer TJ et al (2015) Efficacy and safety of tanezumab monotherapy or combined with non-steroidal anti-inflammatory drugs in the treatment of knee or hip osteoarthritis pain. Ann Rheum Dis 74(6): 1202

58. Hochberg M et al (2019) Subcutaneous tanezumab vs NSAID for the treatment of osteoarthritis: efficacy and general safety results from a randomized, double-blind, active-controlled, 80-week, phase-3 study [abstract]. Arthritis Rheumatol 71(suppl 10). http://acrabstracts.org/abstract/subcutaneous-tanezumabvs-nsaid-for-the-treatment-of-osteoarthritis-efficacy-and-gener al-safety-results-from-a-randomized-double-blind-active-contr olled-80-week-phase-3-study/. Accessed Dec 242019

59. Dakin P et al (2019) The efficacy, tolerability and joint safety of fasinumab in osteoarthritis pain: a phase IIb/III double-blind, placebo-controlled, randomized clinical trial. Arthritis Rheumatol 71(11):1824-1834

60. Hochberg MC (2015) Serious joint-related adverse events in randomized controlled trials of anti-nerve growth factor monoclonal antibodies. Osteoarthr Cartil 23:S18-S21

61. Hochberg MC et al (2015) When is osteonecrosis not osteonecrosis?: Adjudication of reported serious adverse joint events in the tanezumab clinical development program. Arthritis Rheumatol 68(2):382-391

62. Miller RE, Block JA, Malfait AM (2018) What is new in pain modification in osteoarthritis? Rheumatology 57((suppl_4)):iv99-iv107

63. Hochberg M et al (2019) Subcutaneous tanezumab versus NSAID for the treatment of osteoarthritis: joint safety events in a randomized, double-blind, active-controlled, 80-week, phase-3 study [abstract]. Arthritis Rheumatol 71(suppl 10). http://acrab stracts.org/abstract/subcutaneous-tanezumab-versus-nsaid-forthe-treatment-of-osteoarthritis-joint-safety-events-in-a-rando mized-double-blind-active-controlled-80-week-phase-3-study/. Accessed Dec 242019.

64. da Costa BR, Hari R, Jüni P (2016) Intra-articular corticosteroids for osteoarthritis of the knee. JAMA 316(24):2671-2672

65. Arroll B, Goodyear-Smith F (2004) Corticosteroid injections for osteoarthritis of the knee: meta-analysis. BMJ 328(7444):869-869

66. Bodick N et al (2015) An intra-articular, extended-release formulation of triamcinolone acetonide prolongs and amplifies analgesic effect in patients with osteoarthritis of the knee: a randomized clinical trial. J Bone Jt Surg Am 97(11):877-888

67. Conaghan PG et al (2018) Brief report: a phase IIb trial of a novel extended-release microsphere formulation of triamcinolone acetonide for intraarticular injection in knee osteoarthritis. Arthritis Rheumatol 70(2):204-211

68. Conaghan PG et al (2018) Effects of a single intra-articular injection of a microsphere formulation of triamcinolone acetonide on knee osteoarthritis pain: a double-blinded, randomized, placebo-controlled, multinational study. JBJS 100(8):666-677

69. Kraus VB et al (2018) Synovial and systemic pharmacokinetics (PK) of triamcinolone acetonide (TA) following intraarticular (IA) injection of an extended-release microspherebased formulation (FX006) or standard crystalline suspension in patients with knee osteoarthritis (OA). Osteoarthr Cartil 26(1):34-42

70. Conaghan PG et al (2018) Triamcinolone acetonide extendedrelease injectable suspension (TA-ER) is associated with reduced blood glucose elevation vs. standard triamcinolone in type 2 diabetes mellitus patients with knee osteoarthritis: a randomized, blinded, parallel-group study. Osteoarthr Cartil 26:S230

71. Haar D et al (1993) A double-blind comparative study of hydroxychloroquine and dapsone, alone and in combination, in rheumatoid arthritis. Scand J Rheumatol 22(3):113-118

72. Clark P et al (1993) Hydroxychloroquine compared with placebo in rheumatoid arthritis: a randomized, controlled trial. Ann Intern Med 119(11):1067-1071

73. Kyburz D, Brentano F, Gay S (2006) Mode of action of hydroxychloroquine in RA- evidence of an inhibitory effect on toll-like receptor signaling. Nat Clin Pract Rheumatol 2:458

74. Kim HA et al (2006) The catabolic pathway mediated by Tolllike receptors in human osteoarthritic chondrocytes. Arthritis Rheum 54(7):2152-2163

75. Sillat $\mathrm{T}$ et al (2013) Toll-like receptors in human chondrocytes and osteoarthritic cartilage. Acta Orthop 84(6):585-592 
76. Keen HI et al (2008) An ultrasonographic study of osteoarthritis of the hand: synovitis and its relationship to structural pathology and symptoms. Arthritis Care Res 59(12):1756-1763

77. Vlychou M et al (2009) Ultrasonographic evidence of inflammation is frequent in hands of patients with erosive osteoarthritis. Osteoarthr Cartil 17(10):1283-1287

78. Bryant LR, des Rosier KF, Carpenter MT (1995) Hydroxychloroquine in the treatment of erosive osteoarthritis. J Rheumatol 22(8):1527-1531

79. Punzi L et al (1996) Soluble interleukin 2 receptors and treatment with hydroxychloroquine in erosive osteoarthritis. J Rheumatol 23(8):1477-1478

80. Kingsbury SR et al (2018) Hydroxychloroquine effectiveness in reducing symptoms of hand osteoarthritis: a randomized trial. Ann Intern Med 168(6):385-395

81. Lee W et al (2017) Efficacy of hydroxychloroquine in hand osteoarthritis: a randomized, double-blind, placebo-controlled trial. Arthritis Care Res 70(9):1320-1325

82. Wojdasiewicz P, Poniatowski LA, Szukiewicz D (2014) The role of inflammatory and anti-inflammatory cytokines in the pathogenesis of osteoarthritis. Mediat Inflamm 2014:561459

83. Verbruggen $\mathrm{G}$ et al (2012) Tumour necrosis factor blockade for the treatment of erosive osteoarthritis of the interphalangeal finger joints: a double blind, randomised trial on structure modification. Ann Rheum Dis 71(6):891

84. Chevalier $X$ et al (2015) Adalimumab in patients with hand osteoarthritis refractory to analgesics and NSAIDs: a randomised, multicentre, double-blind, placebo-controlled trial. Ann Rheum Dis 74(9):1697-1705

85. Aitken D et al (2018) A randomised double-blind placebocontrolled crossover trial of HUMira (adalimumab) for erosive hand OsteoaRthritis-the HUMOR trial. Osteoarthr Cartil 26(7):880-887

86. Kloppenburg $\mathrm{M}$ et al (2018) Etanercept in patients with inflammatory hand osteoarthritis (EHOA): a multicentre, randomised, double-blind, placebo-controlled trial. Ann Rheum Dis 77(12): 1757

87. Smith MD et al (1997) Synovial membrane inflammation and cytokine production in patients with early osteoarthritis. J Rheumatol 24:365-371

88. Furuzawa-Carballeda J, Macip-Rodriguez PM, Cabral AR (2008) Osteoarthritis and rheumatoid arthritis pannus have similar qualitative metabolic characteristics and pro-inflammatory cytokine response. Clin Exp Rheumatol 26:554-560

89. Goldring MB (2001) Anticytokine therapy for osteoarthritis. Expert Opin Biol Ther 1(5):817-829

90. Caron JP et al (1996) Chondroprotective effect of intraarticular injections of interleukin-1 receptor antagonist in experimental osteoarthritis. Suppression of collagenase-1 expression. Arthritis Rheum 39(9):1535-1544

91. Pelletier J-P et al (2005) In vivo suppression of early experimental osteoarthritis by interleukin-1 receptor antagonist using gene therapy. Arthritis Rheum 40(6):1012-1019

92. Fernandes J et al (1999) In vivo transfer of interleukin-1 receptor antagonist gene in osteoarthritic rabbit knee joints : prevention of osteoarthritis progression. Am J Pathol 154(4):1159-1169

93. Frisbie DD et al (2002) Treatment of experimental equine osteoarthritis by in vivo delivery of the equine interleukin-1 receptor antagonist gene. Gene Ther 9:12
94. Chevalier X et al (2009) Intraarticular injection of anakinra in osteoarthritis of the knee: A multicenter, randomized, double-blind, placebo-controlled study. Arthritis Care Res 61(3):344-352

95. Lacy SE et al (2015) Generation and characterization of ABT981, a dual variable domain immunoglobulin (DVD- $\operatorname{Ig}(\mathrm{TM})$ ) molecule that specifically and potently neutralizes both IL-1 $\alpha$ and IL-1 $\beta$. mAbs 7(3):605-619

96. Fleischmann R et al (2018) SAT0575 Safety and efficacy of lutikizumab (ABT-981), an anti-interleukin-1 alpha/beta dual variable domain (DVD) immunoglobulin, in subjects with knee osteoarthritis: results from the randomised, double-blind, placebo-controlled, parallel-group phase 2 trial. Ann Rheum Dis 77(Suppl 2):1141

97. Kloppenburg M et al (2017) OP0168 A phase 2a, placebocontrolled, randomized study of ABT-981, an anti-interleukin1ALPHA and -1BETA dual variable domain immunoglobulin, to treat erosive hand osteoarthritis (EHOA). Ann Rheum Dis 76(Suppl 2):122

98. Cheleschi S et al (2015) Possible chondroprotective effect of canakinumab: an in vitro study on human osteoarthritic chondrocytes. Cytokine 71(2):165-172

99. Ridker PM et al (2017) Antiinflammatory therapy with canakinumab for atherosclerotic disease. N Engl J Med 377(12):1119-1131

100. Schieker $\mathrm{M}$ et al (2018) Interleukin-1 $\beta$ inhibition with canakinumab associates with reduced rates of total hip and knee replacement (THR/TKR) and osteoarthritis (OA) symptoms: exploratory results from the canakinumab anti-inflammatory thrombosis outcomes study (CANTOS) [abstract]. Arthritis Rheumatol 70(suppl 10). http://acrabstracts.org/abstract/inter leukin- $1 \%$ ce\%b2-inhibition-with-canakinumab-associates-withreduced-rates-of-total-hip-and-knee-replacement-thr-tkr-andosteoarthritis-oa-symptoms-exploratory-results-from-the-canak inumab-ant/. Accessed Dec 242019

101. Kingsbury SR et al (2018) Significant pain reduction with oral methotrexate in knee osteoarthritis; results from a randomised controlled phase III trial of treatment effectiveness [abstract]. Arthritis Rheumatol 70(suppl 10). http://acrabstracts.org/abstr act/significant-pain-reduction-with-oral-methotrexate-in-kneeosteoarthritis-results-from-a-randomised-controlled-phase-iiitrial-of-treatment-effectiveness/. Accessed Dec 242019

102. Ferrero $\mathrm{S}$ et al (2018) Methotrexate in patients with hand erosive osteoarthritis refractory to usual treatments: a randomized, double-blind, placebo-controlled trial [abstract]. Arthritis Rheumatol 71(suppl 10). http://acrabstracts.org/abstract/methotrexatein-patients-with-hand-erosive-osteoarthritis-refractory-to-usual -treatments-a-randomized-double-blind-placebo-controlled-trial I. Accessed Dec 242019

Publisher's Note Springer Nature remains neutral with regard to jurisdictional claims in published maps and institutional affiliations. 